\title{
The Effect of Tissue-Mimicking Phantom Compressibility on Magnetic Hyperthermia
}

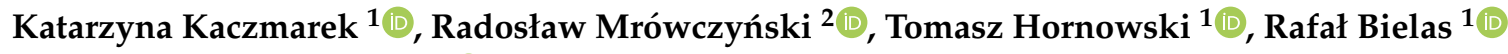 \\ and Arkadiusz Józefczak ${ }^{1, * \mathbb{D}}$ \\ 1 Institute of Acoustics, Faculty of Physics, Adam Mickiewicz University, Uniwersytetu Poznańskiego 2, \\ 61-614 Poznań, Poland; katarzyna.kaczmarek@amu.edu.pl (K.K.); hornaku@amu.edu.pl (T.H.); \\ rafal.bielas@amu.edu.pl (R.B.) \\ 2 NanoBioMedical Centre, Adam Mickiewicz University, Wszechnicy Piastowskiej 3, 61-614 Poznań, Poland; \\ radoslaw.mrowczynski@amu.edu.pl \\ * Correspondence: aras@amu.edu.pl
}

Received: 17 April 2019; Accepted: 23 May 2019; Published: 25 May 2019

check for

\begin{abstract}
During hyperthermia, magnetite nanoparticles placed in an AC magnetic field become a source of heat. It has been shown that in fluid suspensions, magnetic particles move freely and generate heat easily. However, in tissues of different mechanical properties, nanoparticle movement is limited and leads to a small temperature rise in tissue. Therefore, it is crucial to conduct magnetic hyperthermia experiments in similar conditions to the human body. The effect of tissue-mimicking phantom compressibility on the effectiveness of magnetic hyperthermia was investigated on agar phantoms. Single and cluster nanoparticles were synthesized and used as magnetic materials. The prepared magnetic materials were characterized by transmission electron microscopy (TEM), and zeta potential measurements. Results show that tissue-mimicking phantom compressibility decreases with the concentration of agar. Moreover, the lower the compressibility, the lower the thermal effect of magnetic hyperthermia. Specific absorption rate (SAR) values also proved our assumption that tissue-mimicking phantom compressibility affects magnetic losses in the alternating magnetic field (AMF).
\end{abstract}

Keywords: magnetic nanoparticles; magnetic hyperthermia; Brown relaxation; compressibility; tissue-mimicking phantoms

\section{Introduction}

Iron oxide nanoparticles in tumor tissues can act as a heat source because of their intrinsic magnetic features and nanosize. Once tissue with embedded nanoparticles is placed in an alternating magnetic field, the energy from the magnetic field is transformed to heat. Heat transferred from the nanoparticles to the tissue increases its temperature. In the case of superparamagnetic particles, the major heating mechanism is relaxation. The magnetic moment of magnetic nanoparticles tends to align with the direction of the magnetic field through the movement of the magnetic moment within a nanoparticle (Néel relaxation) or the rotation of the whole nanoparticle (Brown relaxation) [1-5]. Very high specific absorption rate (SAR) values of nanoparticle-based magnetic hyperthermia have been obtained for many years. Most research focuses on the development (synthesis and production) of new types of nanoparticles with different shapes, grain and hydrodynamic sizes, magnetic properties, and biocompatible surfactant layers. However, the parameters of nanoparticles that are mentioned are not the only factors influencing the effectiveness of hyperthermia. The heating effect also strongly depends on the medium in which the nanoparticles have to act. It has been shown that magnetic heating works very well in aqueous suspensions $[1,2,5]$. However, high heating power in aqueous suspensions may 
not translate into efficient heating in a cellular environment [6]. In magnetic fluids, particles move freely, whereas in tissue their movement may be limited due to interaction with cells, which alters the particle magnetic relaxation. Various tissues and tumors have different mechanical properties that change as the tumor grows [7]. Solid tumors during growth undergo stiffening and, in general, exhibit higher stiffness and lower elastic moduli than their host's normal tissues [8-10]. Mechanical properties play a crucial role in the growth, development, and therapeutics of tumors [9]. Tumor rigidity may compromise the efficacy of magnetic hyperthermia. Therefore, it is important to understand the influence of mechanical properties on magnetic thermal therapy. Magnetic hyperthermia experiments with nanoparticles should be performed in materials with different elastic properties. To test this phenomenon, tissue-mimicking phantoms (hydrogel) may be used. Hydrogels are commonly used in biomedical applications because of their physical properties, which are similar to those of living tissue.

Various phantoms suitable for hyperthermia studies have been produced in many laboratories over the last decade [11-13]. Agar-based tissue-mimicking materials are specially developed systems that mimic the physical properties of various human tissues. They have been invaluable for developing and testing the thermal effects of hyperthermia [14]. Agar-based thermo-reversible gels consist of thick bundles of agar chains linked by hydrogen bonds, with pores holding water. The mechanical properties of agar-gel to a large extent depend on the mesh-size network (pore size), which is related to agar concentration. With an increase in agar concentration, the pore size decreases due to an increased rate of nucleation and closer packing of the chains. The pore size increases with the setting temperature due to melting of the weak junctions [15]. Because a higher agar concentration produces noticeably stiffer materials, it is reasonable to expect that in phantoms it will correspond to a higher bulk modulus. Stiffness differences result primarily from differences in dry-weight agar concentration [16].

The mechanical properties of phantoms may be evaluated by an acoustic technique that allows one to measure the compressibility of the phantom. In general, ultrasound velocity depends on the bulk modulus $K$ and shear modulus $G$ and can be expressed as $c=\left(\left(K+\frac{4}{3} G\right) / \rho\right)^{1 / 2}$, where $\rho$ is the density of a medium. For fluids, the shear modulus $G$ equals zero. For agar phantoms consisting mainly of water, the shear modulus $G$ is six orders of magnitude smaller than $K$ [17]; therefore, we considered it negligible for our study. When the shear modulus is negligible, we can use a sound velocity equation in the form $c=\left(\rho \beta_{s}\right)^{-1 / 2}$ to estimate adiabatic compressibility $\beta_{s}$. Adiabatic compressibility is an inverse of the bulk modulus $K$. Therefore, ultrasound velocity measurements offer a favorable method for determining the compressibility and bulk modulus of phantoms.

In this study, we analyzed how mechanical properties, such as compressibility, affect magnetic losses in the alternating magnetic field. Magnetic nanoparticles and colloidal clusters composed of multiple magnetic nanoparticles have shown great potential in hyperthermia heating, so single and cluster nanoparticles were synthesized and used as magnetic materials. The effect was examined in particle suspension (magnetic fluid) and ferrogel with various values of compressibility. We demonstrated that an environment in which nanoparticles are embedded affects the temperature increase during magnetic hyperthermia.

\section{Materials and Methods}

\subsection{Magnetic Particles}

Two types of magnetic materials were synthetized to be utilized in our experiments, single nanoparticles (SNPs) and nanosphere cluster nanoparticles (CNPs) composed of small magnetite nanograins.

The reagents used in this work, purchased from Sigma-Aldrich (Hong Kong, China), were iron(III) chloride hexahydrate, iron(II) chloride tetrahydrate, ammonium solution (25\%), citric acid, sodium acetate, sodium acrylate, ethylene glycol, and diethylene glycol.

In order to obtain SNPs, $\mathrm{FeCl}_{2} \cdot 4 \mathrm{H}_{2} \mathrm{O}(1.7 \mathrm{~g}, 8.57 \mathrm{mmol})$ and $\mathrm{FeCl}_{3} \cdot 6 \mathrm{H}_{2} \mathrm{O}(4.7 \mathrm{~g}, 16.2 \mathrm{mmol})$ were dissolved in $80 \mathrm{~mL}$ of deionized water [18]. Then, the solution was degassed with nitrogen and heated 
up to $90{ }^{\circ} \mathrm{C}$, followed by the addition of $20 \mathrm{~mL}$ of $25 \%$ ammonia aqua. The heating continued for $30 \mathrm{~min}$, and then $8 \mathrm{~mL}$ of $20 \%$ citric acid was added. The mixture was kept for $1.5 \mathrm{~h}$ at $90^{\circ} \mathrm{C}$. Finally, the magnetic nanoparticles were collected by an external magnetic field and rinsed with deionized water.

The CNPs were obtained according to Xuan et al. [19]. Briefly, sodium acrylate (1.5 g, $16 \mathrm{mmol})$, sodium acetate $(1.5 \mathrm{~g}, 18.3 \mathrm{mmol})$ and $\mathrm{FeCl}_{3} \cdot 6 \mathrm{H}_{2} \mathrm{O}(0.54 \mathrm{~g}, 2 \mathrm{mmol})$ were stirred in the mixture of ethylene glycol and diethylene glycol until all compounds dissolved. Next, the mixture was transferred to the Teflon autoclave and kept at $200{ }^{\circ} \mathrm{C}$ for $10 \mathrm{~h}$. The mixture was cooled down and particles were collected by centrifugation and washed with EtOH. The obtained material was dried in a vacuum. Finally, the particles were redispersed in water at a concentration of $1 \mathrm{mg} / \mathrm{mL}$.

Morphology of the SNPs and CNPs was determined by transmission electron microscopy (TEM). The TEM images were recorded on a JEM-1400 microscope made by JEOL-1400 (Tokyo, Japan) with an accelerating voltage of $120 \mathrm{kV}$. Samples were dropped cast on a copper grid (Formvar/Carbon 200 mesh made by TedPella (USA)) after 5-15 min of sonication and dried in a vacuum desiccator. For SNPs, TEM revealed a spherical shape of between 8 and $14 \mathrm{~nm}$ (Figure 1a). Zeta potential measurements were determined by Zetasizer Nano ZS made by Malvern Instruments Ltd. (Malvern, United Kingdom).

(a)

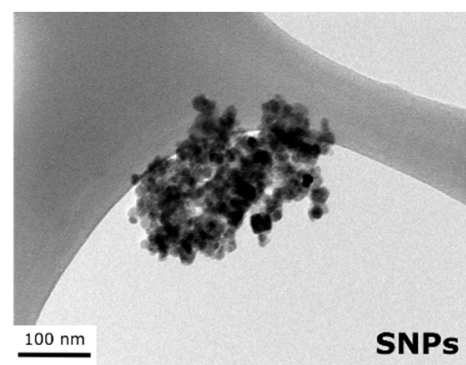

(c)

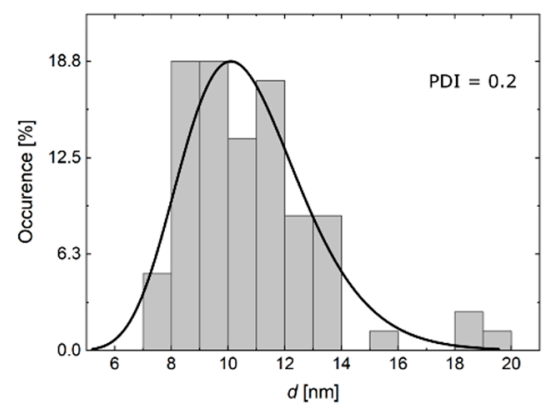

(e)

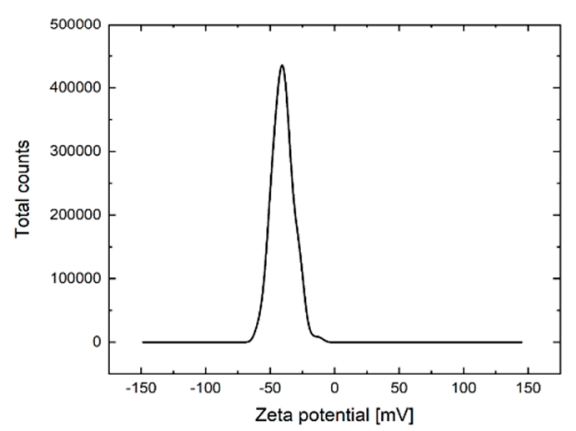

(b)

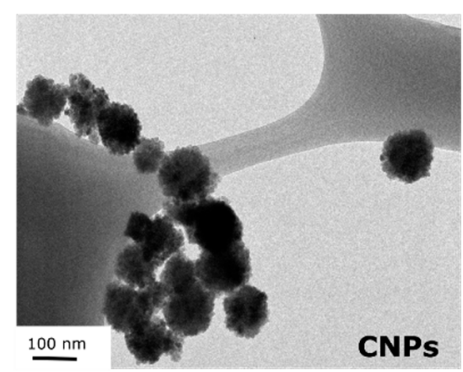

(d)

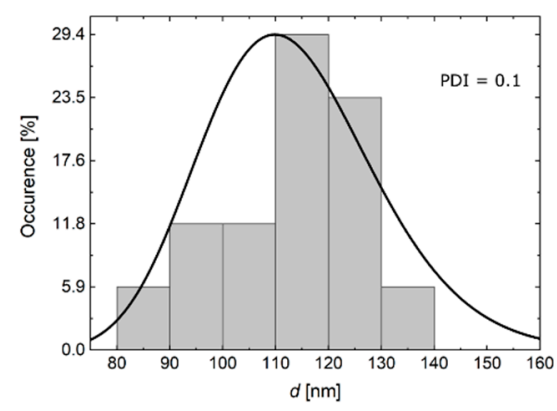

(f)

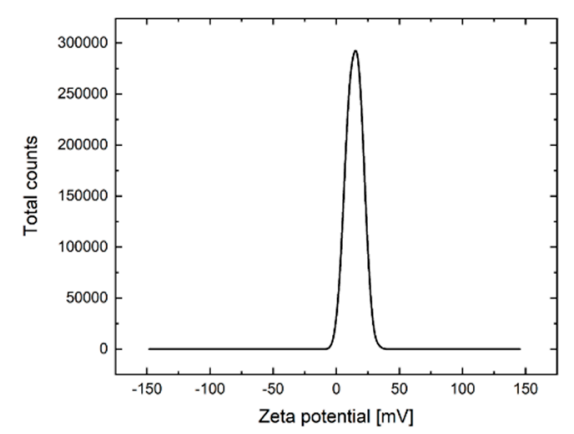

Figure 1. The characterization of nanoparticles: transmission electron microscopy (TEM) micrograph for (a) single nanoparticles (SNPs), (b) cluster nanoparticles (CNPs). Particle size distribution from TEM with calculated polydispersity index (PDI) for (c) SNPs and (d) CNPs. Zeta potential of (e) SNP water suspension and (f) CNP water suspension.

The electric charges on a nanoparticle surface play an important role in the physical stability of nanoparticle-based suspensions. Nanoparticles with zeta potentials values greater than $+30 \mathrm{mV}$ 
or more negative than $-30 \mathrm{mV}$ repel each other and show no tendency to aggregate, thus affording high stability in water [20]. Moreover, the nanoparticle tendency to agglomerate, dependent on surface charges, may affect the hyperthermic effect. We concluded that the obtained SNPs exhibit a strongly negative charge, i.e., $-40 \mathrm{mV}$, which is supposed to assure the colloidal stability of the medium. Single nanoparticles were more stable than CNPs; however, they still had a small tendency to aggregate.

To characterize CNPs, the same techniques were used. The TEM images showed that CNP sizes were between 110 and $130 \mathrm{~nm}$ (Figure 1b). The zeta potential of CNPs showed a slightly positive charge of around $+16 \mathrm{mV}$ (Figure 1f). A slightly positive charge was responsible for their lower stability compared to SNPs.

\subsection{Tissue-Mimicking Phantoms}

In our study, all experiments were performed on agar-based phantoms. Agar consists of a mixture of agarose (the predominant component) and agaropectin [21]. The gelation mechanism is explained as a nucleation and growth process [15]. Three stages can be identified: induction, gelation, and pseudo-equilibrium. Upon cooling down, liquid-liquid phase separation occurs with the formation of nuclei composed of polymer-rich phases. During this induction stage, the sample remains in the sol state. As gelation progresses, the nuclei grow and form a network of polymer-rich phases. The polymer-rich phases tend to coagulate so as to minimize the interface between the polymer-rich and polymer-poor phases, thereby reducing the interfacial free energy. The rigidity of the agarose chains and the aggregation of the chains within the polymer-rich phase prevents large-scale coagulation. Local merging of the polymer-rich phases leads to continuously increasing pore size. In the later part of the gelation stage, most of the agarose chains are immobilized in the fibrous junctions, and local coagulation becomes more difficult [15].

For our experiments, we prepared agar phantoms doped with SNPs or CNPs. The weight concentration of the agar in the phantoms was $3 \%$ and $7 \%(w / w)$, whereas the concentration of the magnetic nanomaterial was $1 \%(w / w)$. The compressibilities $\beta_{s}$ for $3 \%$ and $7 \%$ agar phantom were $4.925 \times 10^{-10} \mathrm{~m}^{2} / \mathrm{N}$ and $4.491 \times 10^{-10} \mathrm{~m}^{2} / \mathrm{N}$. The phantom had a cylindrical shape with a diameter of $3 \mathrm{~cm}$ and a height of approximately $2.5 \mathrm{~cm}$. The employed agar powder is classified by the company Sigma-Aldrich as a plate count agar (product number: 88588).

\subsection{Measurement Setup}

\subsubsection{Ultrasonic Wave Velocity Measurements}

To measure the acoustic velocity, an ultrasonic insertion technique [22] was used. This technique is a relative measurement method of the transmission of longitudinal ultrasonic waves through gel embedded in an aqueous environment [22]. It is based on the measurement of a transit time of a pulse between a pair of transmit and receive transducers. The experimental setup is presented in Figure 2. The transducer operated in transmission mode and was driven by an Optel Pulser/Receiver Card 01/100, which provided a unipolar spike pulse with an amplitude of $360 \mathrm{~V}$ and a fall time better than $20 \mathrm{~ns}$. The received signal was sampled at a rate of $100 \mathrm{MS} / \mathrm{s}$ and recorded. The ultrasonic velocities in the samples were determined from the temporal shift $\Delta t=t_{w}-t_{p}$ between the pulse transit times $t_{w}$ without the phantom and $t_{p}$ with the phantom [23]. The velocity of an ultrasonic wave can be calculated as

$$
c=\left(\frac{1}{c_{w}}-\frac{\Delta t}{l}\right)^{-1}
$$

where $c_{w}$ is the acoustic velocity in water and $l$ the thickness of the gel sample. 


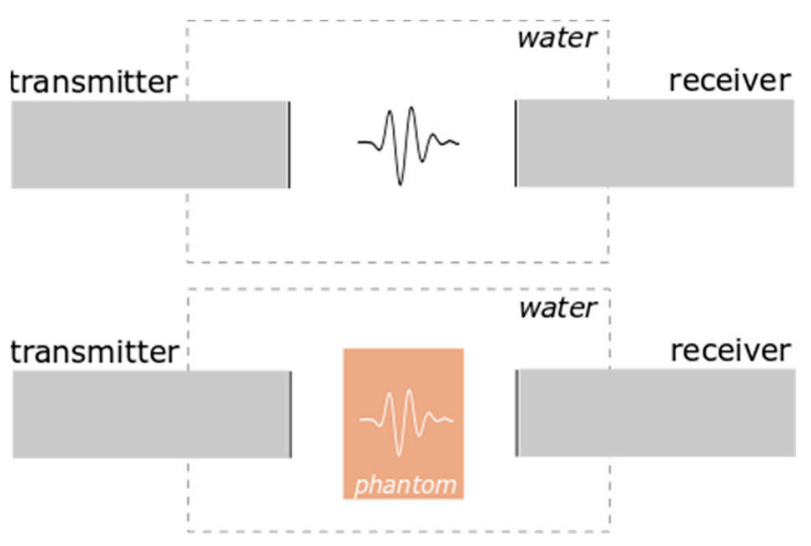

Figure 2. Experimental setup for ultrasonic wave velocity measurements.

During ultrasonic wave velocity measurements, tissue-mimicking phantoms doped with SNPs or CNPs were immersed in distilled water and placed between transmitter and receiver. For measurement of SNP and CNP suspension, the measuring chamber was filled with them. The resulting sound velocities $c$ are presented in Table 1.

Table 1. Sound velocity, density, bulk modulus and compressibility of agar phantom doped with SNPs/CNPs with different $\phi_{a}$ agar concentrations.

\begin{tabular}{cccccc}
\hline $\begin{array}{c}\text { Agar Concentration } \\
\boldsymbol{\phi}_{\boldsymbol{a}}[\mathbf{\%}]\end{array}$ & $\begin{array}{c}\text { Type of } \\
\text { Nanomaterials }\end{array}$ & $\begin{array}{c}\text { Sound } \\
\text { Velocity }[\mathbf{m} / \mathbf{s}]\end{array}$ & $\begin{array}{c}\text { Density } \\
{\left[\mathbf{k g} / \mathbf{m}^{\mathbf{3}}\right]}\end{array}$ & $\begin{array}{c}\text { Bulk Modulus } \\
{[\mathbf{G P a}]}\end{array}$ & $\begin{array}{c}\text { Compressibility } \\
\left.\boldsymbol{\beta}_{\mathbf{s}}\left[\mathbf{m}^{\mathbf{2}} / \mathbf{N}\right)\right]\end{array}$ \\
\hline 0 & SNPs & 1479.29 & 987 & 2.160 & $4.630 \times 10^{-10}$ \\
3 & SNPs & 1498.30 & 1010 & 2.267 & $4.411 \times 10^{-10}$ \\
7 & SNPs & 1509.00 & 1022 & 2.327 & $4.297 \times 10^{-10}$ \\
0 & CNPs & 1483.68 & 995 & 2.190 & $4.566 \times 10^{-10}$ \\
3 & CNPs & 1503.60 & 1010 & 2.283 & $4.379 \times 10^{-10}$ \\
7 & CNPs & 1507.60 & 1024 & 2.328 & $4.297 \times 10^{-10}$ \\
\hline
\end{tabular}

\subsubsection{Density Measurements}

The phantom density was established according to Archimedes' principle. The phantoms were first weighted in air and then weighted again after submersion in distilled water. Density was calculated using the formula $\rho=\frac{m_{a}}{\left(m_{a}-m_{a w}\right)} \rho_{w}$, where $m_{a}$ is the mass of the phantom in air, $m_{a w}$ is the mass of the phantom submerged in water, and $\rho_{w}$ is the density of distilled water. For precise weighting, analytical laboratory balances (Radwag AS 220/X; Radwag, Radom, Poland), with readability to $1 \mathrm{mg}$, were used.

\subsubsection{Hyperthermia Measurements}

The heating power of magnetic nanomaterials can be calculated using calorimetric measurements. The specific absorption rate (SAR) volume was evaluated to characterize the power deposition in the tissue during the thermal treatment. The SAR value depends on the efficiency of the heat source and the ability of the heated medium to absorb thermal energy. Thus, in the case of magnetic particle heating, the SAR value depends on the concentration of nanoparticles and their ability to rotate (Brown mechanism) in dense material. According to the usual definition, the SAR describes the rate of energy absorption by a material and can be calculated as:

$$
\mathrm{SAR}=c_{p}\left(\frac{\mathrm{d} T}{\mathrm{~d} t}\right)_{t=0}
$$

where $c_{p}$ is the specific heat of the phantom. In practice, obtaining the SAR experimentally involves measurement of the temperature increase following a step-in heating, fitting the resulting data to a linear function in time, and then determining its slope at time zero. 
The compact EASYHEAT (Ambrell Corporation, Scottsville, NY, United States) induction heating system was used as a source for an alternating magnetic field. The induction heating setup consists of a high-frequency power supply that takes the input from the AC line mains. The high-oscillating signal is then fed to a tank circuit that feeds the water-cooled induction heating coil. The high-frequency electric signal generates a high-frequency magnetic field inside the induction coil. The phantom was inserted into a sample cell that was centrally placed and thermally insulated in a water-cooled magnetic induction coil. The frequency of the alternating magnetic field is $356 \mathrm{kHz}$ and the maximal intensity is $16.2 \mathrm{kA} / \mathrm{m}$. The temperature in the phantom during all hyperthermia experiments was measured using a FLUOTEMP temperature sensor system (Photon Control Inc., Burnaby, BC, Canada) with the optic fiber temperature probe (model FTP-NY2). The signal from the probe is unaffected by the magnetic field and other interferences. During experiments, the optical fiber was centrally placed in the phantom. The experimental setup for magnetic heating measurements is presented in Figure 3.

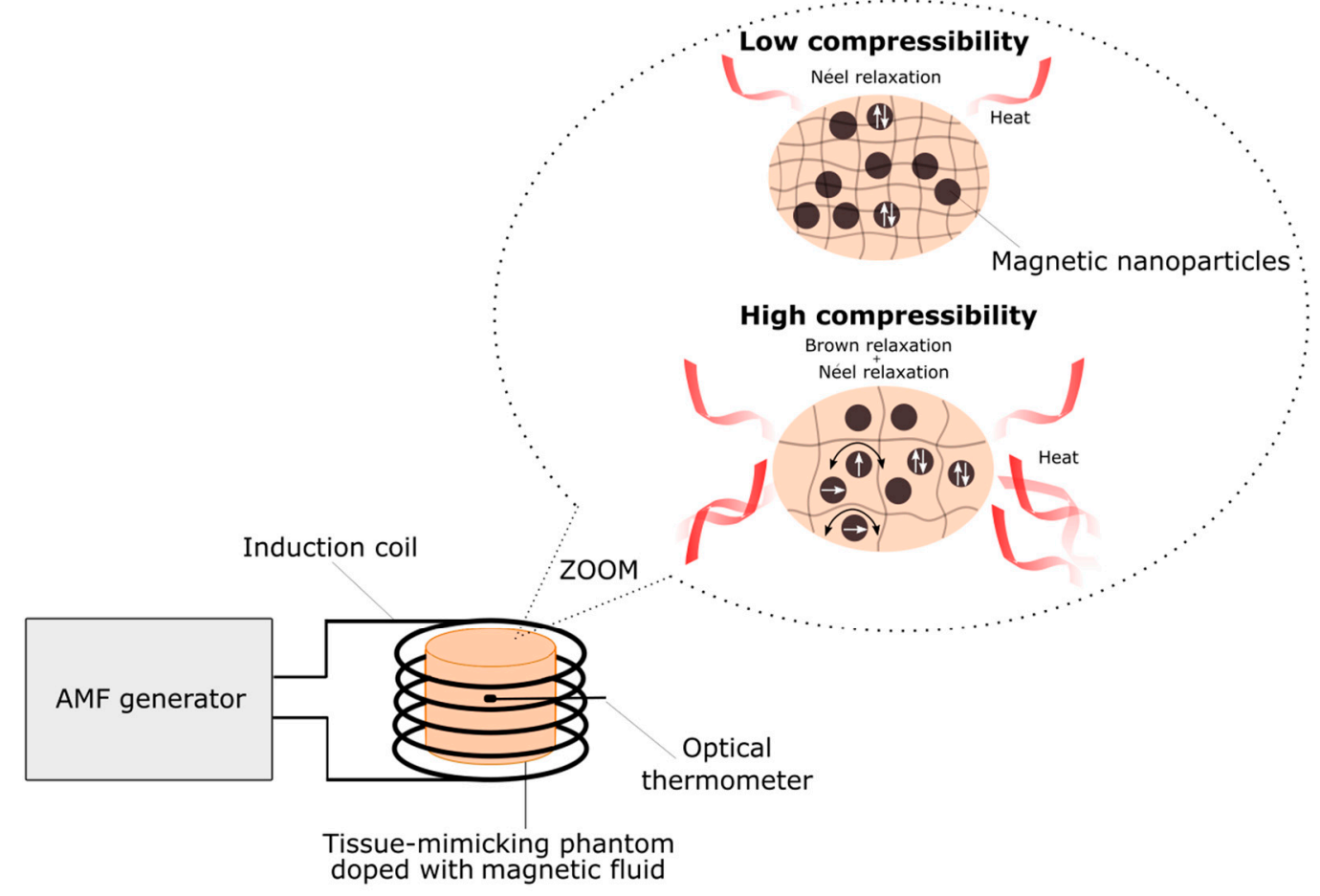

Figure 3. Experimental setup for an alternating magnetic field (AMF) hyperthermia measurements.

\section{Results and Discussion}

\subsection{Elastic Properties}

To conduct all experiments in conditions similar to human tissues of various mechanical properties, we prepared phantoms with different concentrations of agar $\phi_{a}$, resulting in various elastic properties. Based on the experimental results of sound velocity and density measurements, the bulk modulus and compressibility of SNP/CNP water suspension and agar phantoms doped with those magnetic fluids were calculated. The obtained values of sound velocity, density, bulk modulus and compressibility are presented in Table 1.

Tissue-mimicking phantoms with a higher agar concentration $\phi_{a}$ have a higher bulk modulus $K$, which noticeably corresponds to the stiffness of the prepared material. The stiffness of agar phantoms depends heavily on the concentration of agar; therefore, it is appropriate to study in them the effectiveness of hyperthermia as a function of phantom mechanical properties. It can also be seen that density and sound velocity increase with agar concentration $\phi_{a}$ in tissue-mimicking phantoms, while the compressibility decreases. Moreover, the obtained compressibilities for tissue-mimicking phantoms 
doped with magnetic material are lower than the compressibilities for pure agar phantoms that can be found in part 2.2. Interestingly, as shown in Figure 4, with increasing agar concentration $\phi_{a}$ in the phantom, the difference between used magnetic suspensions diminishes. It can be concluded that the stiffness of the material for the $7 \%$ agar phantom is so substantial that the characteristic of the used magnetic material (overall size and type: single, cluster) do not influence phantom compressibility.

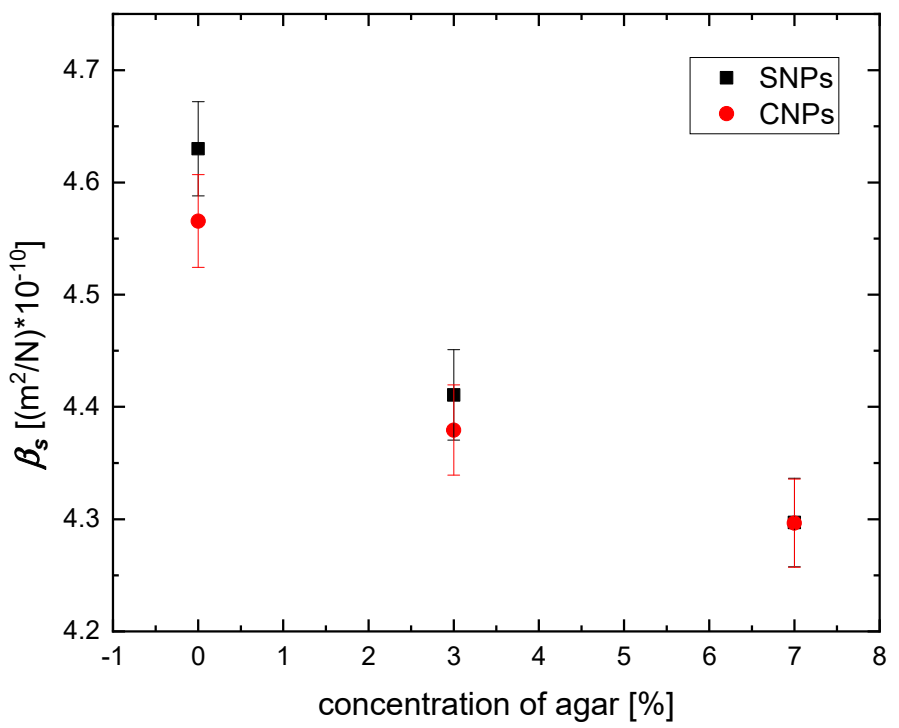

Figure 4. Compressibility of tissue-mimicking phantoms doped with SNPs or CNPs for different concentrations of agar.

\subsection{Magnetic Hyperthermia}

The heating effect of magnetic particles is a result of an absorbing energy from the alternating magnetic field and its conversion into heat. There are three mechanisms responsible for this effect: eddy current losses, hysteresis losses during reversal of magnetization, and relaxation losses accompanying demagnetization. However, to single domain nanoparticles, only the relaxation mechanism applies.

Néel relaxation dominates for small particles (diameter up to $14 \mathrm{~nm}$ ). Brown relaxation dominates for larger sizes (diameter above $14 \mathrm{~nm}$ ) [24]. The distribution curve of nanoparticle size is not narrow. Therefore, it can be concluded that both mechanisms of relaxation will be present during magnetic hyperthermia. As the Brown and Néel processes contributes to the overall heating, the effective relaxation time is given by

$$
\tau_{e f f}=\frac{\tau_{B} \cdot \tau_{N}}{\tau_{B}+\tau_{N}}
$$

where $\tau_{N}$ is Néel relaxation time and $\tau_{B}$ is Brown relaxation time. The Néel relaxation, which does not depend on viscoelastic properties, can be described as

$$
\tau_{N}=\tau_{0} \exp \left(\frac{K V}{k_{B} T}\right)
$$

where $K V$ is the magnetic anisotropy energy barrier, $k_{B}$ is the Boltzmann constant, and $T$ is the temperature.

The Brown relaxation mechanism originates from the rotation of the whole particle in a viscous carrier fluid and is described by a relaxation time $\tau_{B}$, which depends on the hydrodynamic volume $V_{\mathrm{h}}$, shear viscosity $\eta_{\mathrm{s}}$, and temperature $T$

$$
\tau_{B}=\frac{3 \eta_{s} \cdot V_{h}}{k_{B} T} .
$$

In order to set the magnetic moment in the direction of the applied magnetic field, the whole magnetic particle must rotate. The Brown effect in dense materials, such as gels or tissues, is very 
limited due to the fairly fixed position the nanoparticles occupy in the medium. In magnetic fluids, nanoparticles move freely and generate heat easily. To confirm this assumption, magnetic heating experiments as a function of phantom compressibility were conducted.

The results of magnetic heating performed on phantoms were compared to the results performed on SNP and CNP water suspensions (Figures 5 and 6).

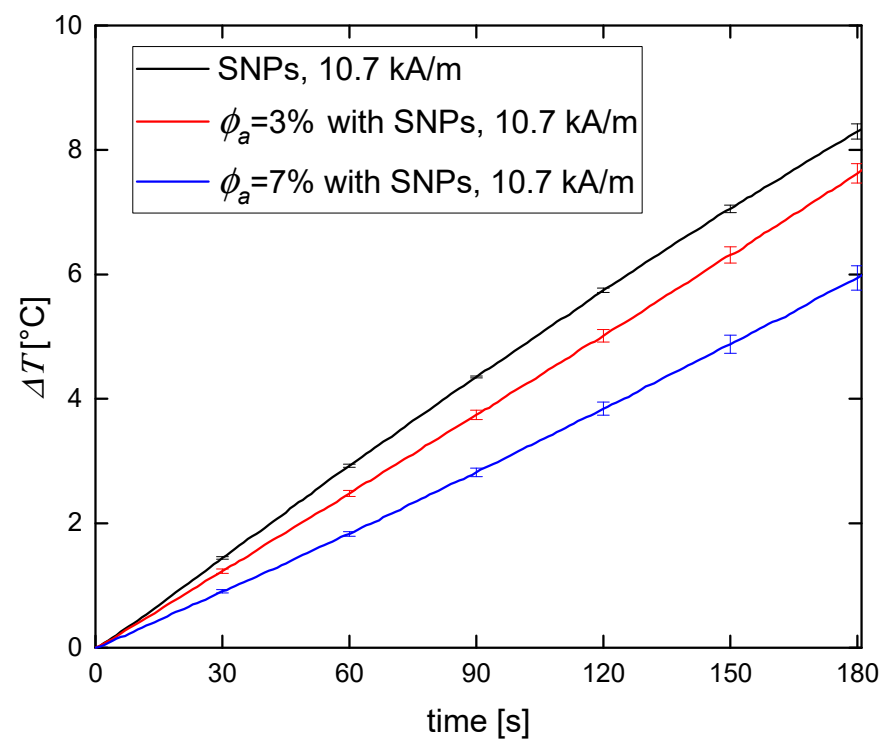

Figure 5. Temperature increase for SNPs suspended in water and embedded in 3\% and $7 \%$ agar phantom for $10.7 \mathrm{kA} / \mathrm{m}$ of the magnetic field.

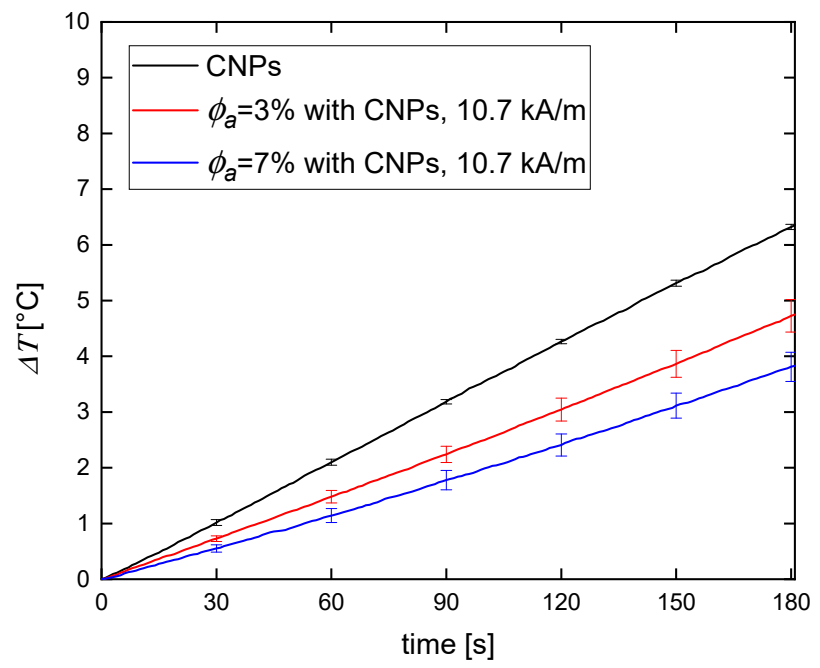

Figure 6. Temperature increase for CNPs suspended in water and embedded in 3\% and 7\% agar phantom for $10.7 \mathrm{kA} / \mathrm{m}$ of the magnetic field.

Regardless of the type of the magnetic material used in the phantom, the temperature increase for $3 \%$ agar phantom was higher than for $7 \%$ agar phantom. In Table 1 we showed that the higher the agar concentration of the phantom, the lower its compressibility. Thus, the observed decrease in magnetic heating efficiency is a consequence of the inhibition of Brown relaxation (physical particle rotation). Néel relaxation (magnetic moment rotation) is independent of the stiffness of the medium in which magnetic particles are embedded, so it does not vary from concentration to concentration. The differences in hyperthermia efficiency between water suspensions of magnetic nanoparticles and tissue-mimicking phantoms were recently reported by Avolio et al. [25]. They proved that when using smaller magnetic nanoparticles (around $10 \mathrm{~nm}$ in size), the obtained efficiency (expressed by 
the SAR value) was comparable for both water suspension and hydrogel doped with nanoparticles. As mentioned above, it is the diameter's range of Néel relaxation domination. Otherwise, for bigger nanoparticles from the range of Brown relaxation, the observable difference between water suspensions and phantoms was indicated.

Our magnetic hyperthermia experiments for CNPs embedded in agar phantoms with various values of stiffness were also performed for several values of magnetic fields: 5.2, 10.7, and $16.2 \mathrm{kA} / \mathrm{m}$. Figure 7 shows that the higher the magnetic field, the higher the temperature increase. These results prove again that agar concentration, used to form tissue-mimicking phantoms, has an impact on the obtained temperature rise. For each experimental line in Figure 7, one can see the differences between $3 \%$ and $7 \%$ agar concentrations.

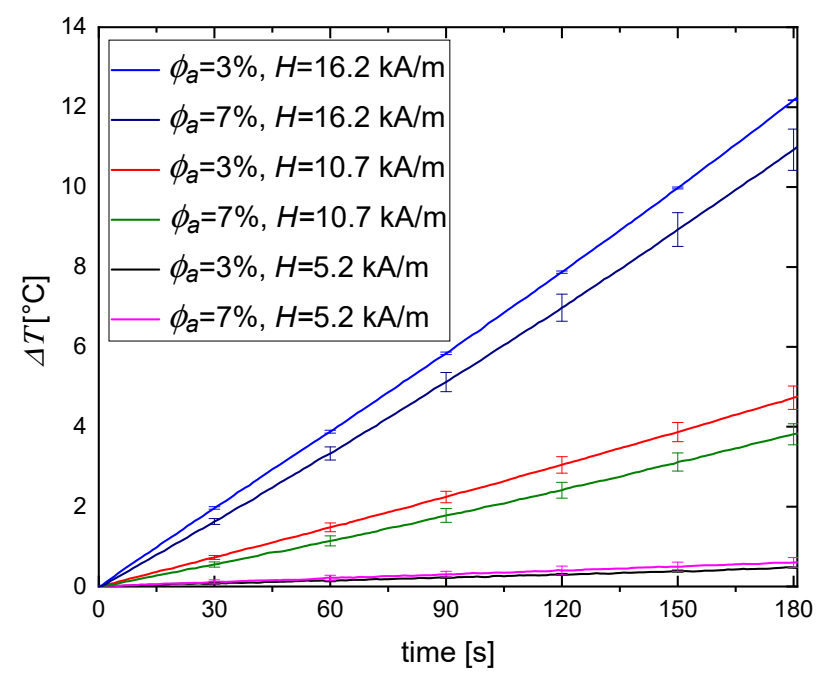

Figure 7. Temperature increase for CNPs embedded in $3 \%$ and $7 \%$ agar phantom during the heating in AMF at 5.2, 10.7, and $16.2 \mathrm{kA} / \mathrm{m}$ strength.

For our experiments, we used two types of magnetic particles: single and cluster nanoparticles. Although the size of a single nanoparticle separated from CNPs has a similar size to SNPs, the overall size of the CNPs is larger, as shown in Figure 1a,b. For that reason, CNPs in an alternating magnetic field move less freely than SNPs, which results in less effective heating than SNPs, regardless of the magnetic field intensity. This trend is especially visible when combining the results for temperature rises in the same magnetic field conditions for both single and cluster nanoparticles (Figure 8). The above conclusions correspond well with the other works on magnetic heating efficiency in hydrogels. Engelmann et al. [26] recently indicated that the macroscopic structure of the gel, in which magnetic nanoparticles are embedded (mesh size), can hinder Brown relaxation for sufficiently large magnetic objects. This effect is not the only one that explains the lower efficiency of magnetic hyperthermia for cluster nanoparticles. It has been reported that the dipole interactions of magnetic nanoparticles in a cluster can also influence heating. When the clusters are isotropic in shape, heating efficiency is lower than that of non-interacting particles despite the cluster's size [27]. Other authors [28] reported that heating efficiency for maghemite clusters $(45-98 \mathrm{~nm})$ in water media is better than for single nanoparticles $(13 \mathrm{~nm})$, due to the fact that the surface of SNPs cools down more rapidly than the surface of CNPs. However, as the authors also indicated, the heating efficiency of clusters decreases with the increase of their size. The highest heating efficiency is for the cluster size around $50 \mathrm{~nm}$, but for the bigger cluster, it clearly starts to decrease. It can be assumed that with a further decrease of CNP size, the same, or even worse, heating effects observed for SNPs can be expected for CNPs. In our work, we used magnetite CNPs with sizes much larger than $50 \mathrm{~nm}(110-130 \mathrm{~nm})$, so we obtained a worse heating effect for clusters than for single nanoparticles. The mentioned phenomena may also play a role, and act collectively, in our experiments. 


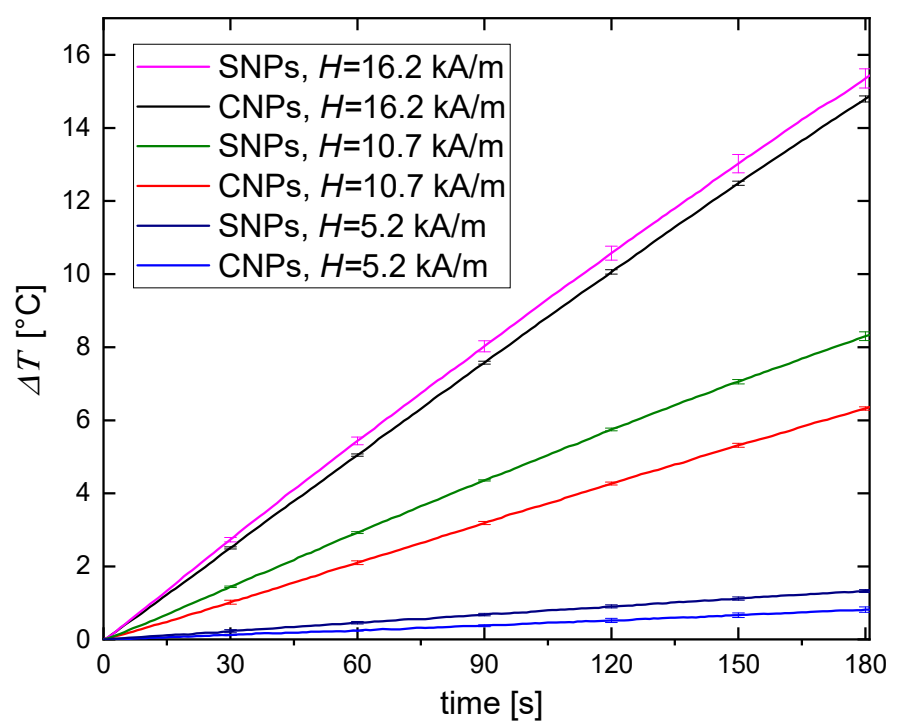

Figure 8. Comparison of temperature increase for SNP and CNP water suspension during heating in AMF at 5.2, 10.7 and $16.2 \mathrm{kA} / \mathrm{m}$ strength.

To characterize the power dissipation in the phantom, during the magnetic hyperthermia experiments, the SAR was evaluated. The SAR value provides information about the efficiency of the heat source (rate of temperature rise) and the ability of the heated medium to absorb thermal energy-see Equation (2). As we proved above, it depends on the nanoparticles' ability to rotate (Brown mechanism). Therefore, we calculated the SAR as a function of phantom compressibility $\beta_{s}$. The results are shown in Figure 9.

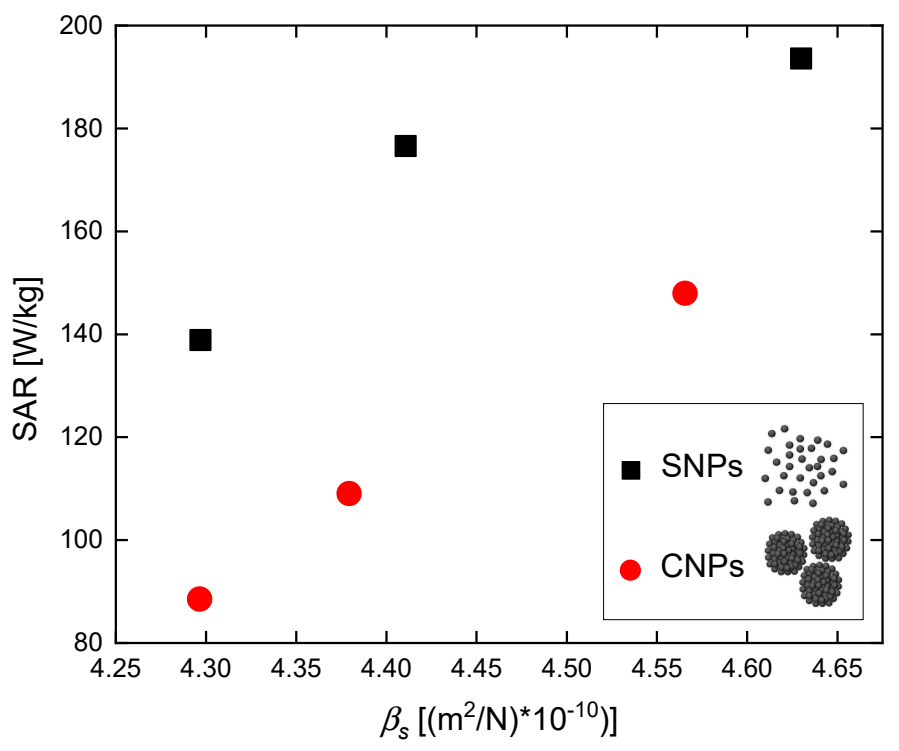

Figure 9. Specific absorption rate in a function of compressibility of tissue-mimicking phantom doped with SNPs or CNPs.

Our calculations show that the SAR values increase with increasing compressibility $\beta_{s}$. This result again proves that tissue-mimicking phantom compressibility affects the effectiveness of the magnetic hyperthermia effect.

The low thermal effect of magnetic hyperthermia obtained in tissues can be improved. Combining magnetic hyperthermia treatment with other therapeutic methods, such as ultrasonic heating, can lead to a more effective output. In our previous work concerning magneto-ultrasonic heating [29], we 
demonstrated that a bimodal stimulation of nanoparticles provides better heating efficiency. Moreover, additional heating caused by ultrasound sonication may increase the phantom pores (as in sonophoresis treatment), allowing nanoparticles to move more freely. In this case, magnetic hyperthermia is improved by unlocking the Brown mechanism.

\section{Conclusions}

Our research showed that in fluid suspensions, magnetic particles move freely and generate heat easily. In tissue-mimicking phantoms, whose mechanical properties are different, nanoparticle movement is limited, and the generated heat is smaller. The firm gel structure of the phantom does not affect the Néel mechanism. However, the higher the agar concentration of the phantom, the lower the compressibility, which leads to an inhibition of Brown relaxation, resulting in less effective magnetic heating. This is independent of the form of the nanoparticles (single vs. cluster). When medical usage of magnetic hyperthermia is considered, deterioration of the heating efficiency of the magnetic fluid, after being introduced into the body, should be considered. Nowadays, awareness of this fact in scientific reports is increasing [25]. Despite the low thermal effect of magnetic hyperthermia, its effectiveness can be improved. Combining magnetic hyperthermia treatment with other therapeutic methods, such as ultrasonic heating, can lead to more effective heating due to the unblocking of the Brown thermal mechanism [29]. Our research also showed that for low compressibility materials (phantoms with high agar concentration), there is no difference in heating efficiency for both types of magnetic materials (single or cluster nanoparticles). However, for materials with higher compressibility (phantoms with low agar concentration), the matter is different. The heating efficiency of single nanoparticles is higher than that of cluster nanoparticles.

Author Contributions: A.J. and K.K. initiated the project and designed the experiments. K.K. and R.M. performed the experiments. K.K., R.B. and T.H. analyzed the data. K.K wrote the first version of the manuscript. All authors took part in discussions towards finalization of the manuscript.

Funding: This work was partly supported by the grants, 2015/17/B/ST7/03566 (OPUS), 2017/27/N/ST7/00201 (PRELUDIUM) and UMO-2014/13/D/ST5/02793, of the Polish National Science Centre.

Acknowledgments: The authors wish to thank Damian Maziukiewicz for laboratory support during the characterization of magnetic materials.

Conflicts of Interest: The authors declare no conflict of interest.

\section{References}

1. Hergt, R.; Hiergeist, R.; Hilger, I.; Kaiser, W.; Lapatnikov, Y.; Margel, S.; Richter, U. Maghemite nanoparticles with very high AC-losses for application in RF-magnetic hyperthermia. J. Magn. Magn. Mater. 2004, 270, 345-357. [CrossRef]

2. Zhang, L.-Y.; Gu, H.-C.; Wang, X.-M. Magnetite ferrofluid with high specific absorption rate for application in hyperthermia. J. Magn. Magn. Mater. 2007, 311, 228-233. [CrossRef]

3. Iglesias, G.; Delgado, A.; Kujda, M.; Ramos-Tejada, M.; Ramos-Tejada, M.D.M. Magnetic hyperthermia with magnetite nanoparticles: Electrostatic and polymeric stabilization. Colloid Polym. Sci. 2016, 294, 1541-1550. [CrossRef]

4. Darwish, M.S. Effect of carriers on heating efficiency of oleic acid-stabilized magnetite nanoparticles. J. Mol. Liq. 2017, 231, 80-85. [CrossRef]

5. Natividad, E.; Andreu, I. Characterization of Magnetic Hyperthermia in Magnetic Nanoparticles. In Magnetic Characterization Techniques for Nanomaterials; Kumar, C.S.S.R., Ed.; Springer: Berlin/Heidelberg, Germany, 2017; pp. 261-303.

6. Espinosa, A.; Di Corato, R.; Kolosnjaj-Tabi, J.; Flaud, P.; Pellegrino, T.; Wilhelm, C. The Duality of Iron Oxide Nanoparticles in Cancer Therapy: Amplification of Heating Efficiency by Magnetic Hyperthermia and Photothermal Bimodal Treatment. ACS Nano 2016, 10, 2436-2446. [CrossRef]

7. Saraf, H.; Ramesh, K.; Lennon, A.; Merkle, A.; Roberts, J. Mechanical properties of soft human tissues under dynamic loading. J. Biomech. 2007, 40, 1960-1967. [CrossRef] 
8. Paszek, M.J.; Zahir, N.; Johnson, K.R.; Lakins, J.N.; Rozenberg, G.I.; Gefen, A.; Reinhart-King, C.A.; Margulies, S.S.; Dembo, M.; Boettiger, D.; et al. Tensional homeostasis and the malignant phenotype. Cancer Cell 2005, 8, 241-254. [CrossRef]

9. Xue, S.-L.; Lin, S.-Z.; Li, B.; Feng, X.-Q. A nonlinear poroelastic theory of solid tumors with glycosaminoglycan swelling. J. Theor. Boil. 2017, 433, 49-56. [CrossRef]

10. Plodinec, M.; Loparic, M.; Monnier, C.A.; Obermann, E.C.; Zanetti-Dällenbach, R.; Oertle, P.; Hyotyla, J.T.; Aebi, U.; Bentires-Alj, M.; Lim, R.Y.H.; et al. The nanomechanical signature of breast cancer. Nat. Nanotechnol. 2012, 7, 757-765. [CrossRef]

11. Bazan, I.; Vazquez, M.; Ramos, A.; Vera, A.; Leija, L. A performance analysis of echographic ultrasonic techniques for non-invasive temperature estimation in hyperthermia range using phantoms with scatterers. Ultrasonics 2009, 49, 358-376. [CrossRef] [PubMed]

12. Miaskowski, A.; Sawicki, B. Magnetic Fluid Hyperthermia Modeling Based on Phantom Measurements and Realistic Breast Model. IEEE Trans. Biomed. Eng. 2013, 60, 1806-1813. [CrossRef] [PubMed]

13. Ma, M.; Zhang, Y.; Gu, N. Estimation the tumor temperature in magnetic nanoparticle hyperthermia by infrared thermography: Phantom and numerical studies. J. Boil. 2018, 76, 89-94. [CrossRef] [PubMed]

14. Singh, H.; Kumar, A.; Singh, V.; Sethi, P.; Singh, M.; Pant, K. Development of tissue-equivalent phantoms for biomedical ultrasonic applications. Int. J. Biomed. Eng. Technol. 2008, 1, 273. [CrossRef]

15. Narayanan, J.; Xiong, J.-Y.; Liu, X.Y. Determination of agarose gel pore size: Absorbance measurements vis a vis other techniques. J. Phys. Conf. Ser. 2006, 28, 83.

16. Ernest, L.M.; Maritza, A.H.; Hairong, S.; Tomy, V.; Gary, R.F. Tissue-mimicking agar/gelatin materials for use in heterogeneous elastography phantoms. Phys. Med. Boil. 2005, 50, 5597.

17. Manickam, K.; Machireddy, R.R.; Seshadri, S. Characterization of biomechanical properties of agar based tissue mimicking phantoms for ultrasound stiffness imaging techniques. J. Mech. Biomed. Mater. 2014, 35, 132-143. [CrossRef] [PubMed]

18. Mrówczyński, R.; Jedrzak, A.; Szutkowski, K.; Grześkowiak, B.F.; Coy, E.; Markiewicz, R.; Jesionowski, T.; Jurga, S. Cyclodextrin-Based Magnetic Nanoparticles for Cancer Therapy. Nanomaterials 2018, 8, 170. [CrossRef]

19. Xuan, S.; Wang, Y.-X.J.; Yu, J.C.; Leung, K.C.-F. Tuning the Grain Size and Particle Size of Superparamagnetic Fe3O4Microparticles. Chem. Mater. 2009, 21, 5079-5087. [CrossRef]

20. Iannazzo, D.; Pistone, A.; Celesti, C.; Triolo, C.; Patané, S.; Giofré, S.V.; Romeo, R.; Ziccarelli, I.; Mancuso, R.; Gabriele, B.; et al. A Smart Nanovector for Cancer Targeted Drug Delivery Based on Graphene Quantum Dots. Nanomaterials 2019, 9, 282. [CrossRef]

21. Armisén, R. International Workshop on Gelidium; Juanes, J.A., Santelices, B., McLachlan, J.L., Eds.; Agar and agarose biotechnological applications; Springer: Dordrecht, The Netherlands, 1991; pp. 157-166.

22. Zell, K.; Sperl, J.I.; Vogel, M.W.; Niessner, R.; Haisch, C. Acoustical properties of selected tissue phantom materials for ultrasound imaging. Phys. Med. Boil. 2007, 52, N475-N484. [CrossRef]

23. Józefczak, A.; Kaczmarek, K.; Kubovčíková, M.; Rozynek, Z.; Hornowski, T. The effect of magnetic nanoparticles on the acoustic properties of tissue-mimicking agar-gel phantoms. J. Magn. Magn. Mater. 2017, 431, 172-175.

24. Deatsch, A.E.; Evans, B.A. Heating efficiency in magnetic nanoparticle hyperthermia. J. Magn. Magn. Mater. 2014, 354, 163-172. [CrossRef]

25. Avolio, M.; Guerrini, A.; Brero, F.; Innocenti, C.; Sangregorio, C.; Cobianchi, M.; Mariani, M.; Orsini, F.; Arosio, P.; Lascialfari, A. In-gel study of the effect of magnetic nanoparticles immobilization on their heating efficiency for application in Magnetic Fluid Hyperthermia. J. Magn. Magn. Mater. 2019, 471, 504-512. [CrossRef]

26. Engelmann, U.M.; Seifert, J.; Mues, B.; Roitsch, S.; Ménager, C.; Schmidt, A.M.; Slabu, I. Heating efficiency of magnetic nanoparticles decreases with gradual immobilization in hydrogels. J. Magn. Magn. Mater. 2019, 471, 486-494. [CrossRef]

27. Fu, R.; Yan, Y.; Roberts, C.; Liu, Z.; Chen, Y. The role of dipole interactions in hyperthermia heating colloidal clusters of densely-packed superparamagnetic nanoparticles. Sci. Rep. 2018, 8, 4704. [CrossRef] [PubMed] 
28. Sakellari, D.; Brintakis, K.; Kostopoulou, A.; Myrovali, E.; Simeonidis, K.; Lappas, A.; Angelakeris, M. Ferrimagnetic nanocrystal assemblies as versatile magnetic particle hyperthermia mediators. Mater. Sci. Eng. C 2016, 58, 187-193. [CrossRef] [PubMed]

29. Kaczmarek, K.; Hornowski, T.; Antal, I.; Timko, M.; Józefczak, A. Magneto-ultrasonic heating with nanoparticles. J. Magn. Magn. Mater. 2019, 474, 400-405. [CrossRef]

(C) 2019 by the authors. Licensee MDPI, Basel, Switzerland. This article is an open access article distributed under the terms and conditions of the Creative Commons Attribution (CC BY) license (http://creativecommons.org/licenses/by/4.0/). 